Article

\title{
Green Synthesis of Smart Metal/Polymer Nanocomposite Particles and Their Tuneable Catalytic Activities
}

\author{
Noel Peter Bengzon Tan, Cheng Hao Lee and Pei Li * \\ Department of Applied Biology and Chemical Technology, The Hong Kong Polytechnic University, Hung Hom, \\ Kowloon, Hong Kong, China; bengzontan@nami.org.hk (N.P.B.T.); chenghao.lee@polyu.edu.hk (C.H.L.) \\ * Correspondence: pei.li@polyu.edu.hk; Tel.: +852-3400-8721 \\ Academic Editor: Haruma Kawaguchi \\ Received: 17 February 2016; Accepted: 17 March 2016; Published: 23 March 2016
}

\begin{abstract}
Herein we report a simple and green synthesis of smart Au and Ag@Au nanocomposite particles using poly(N-isopropylacrylamide)/polyethyleneimine (PNIPAm/PEI) core-shell microgels as dual reductant and templates in an aqueous system. The nanocomposite particles were synthesized through a spontaneous reduction of tetrachloroauric (III) acid to gold nanoparticles at room temperature, and in situ encapsulation and stabilization of the resultant gold nanoparticles (AuNPs) with amine-rich PEI shells. The preformed gold nanoparticles then acted as seed nanoparticles for further generation of Ag@Au bimetallic nanoparticles within the microgel templates at $60^{\circ} \mathrm{C}$. These nanocomposite particles were characterized by TEM, AFM, XPS, UV-vis spectroscopy, zeta-potential, and particle size analysis. The synergistic effects of the smart nanocomposite particles were studied via the reduction of $p$-nitrophenol to $p$-aminophenol. The catalytic performance of the bimetallic Ag@Au nanocomposite particles was 25-fold higher than that of the monometallic Au nanoparticles. Finally, the controllable catalytic activities of the Au@PNIPAm/PEI nanocomposite particles were demonstrated via tuning the solution $\mathrm{pH}$ and temperature.
\end{abstract}

Keywords: gold nanoparticles; silver/gold bimetallic nanoparticles; smart core-shell microgel; metal/polymer nanocomposite; reduction of $p$-nitrophenol

\section{Introduction}

Metal nanoparticles have gained much attention over the past two decades because of their unique properties. Their distinctive size and shape-dependent optical and electronic properties have opened up vast potential applications in various fields [1]. However, metallic nanoparticles have a tendency to aggregate due to their large surface energy, which drives the thermodynamically favored coalescence process. Various types of stabilizing agents have been used to prevent nanoparticles from aggregating. They include ligands [2], surfactants [3,4], polymers [5,6], dendrimers [7], cyclodextrin [8], and microgels [9]. Recently, the use of smart polymer particles to stabilize metallic nanoparticles has received increasing attention because of the synergistic properties of the smart metal/polymer nanocomposite particles, derived from responsive polymer and metallic nanoparticles $[10,11]$. Smart metal/polymer nanocomposite particles of this kind have shown promising potential for use as hybrid materials for biomedical applications [12,13], sensing [14], and catalytic reactions [15,16].

Our group has recently reported a simple and green synthesis of AuNPs/polymer nanocomposite particles through a spontaneous reduction of tetrachloroauric (III) acid and encapsulation of the resultant gold nanoparticles using amine-rich core-shell particles in water $[17,18]$. The high local amine concentration of the polyethyleneimine shell enables effective reduction of gold ions to gold nanoparticles in the absence of any organic solvents, reducing agents, or stabilizers. 
Thus we envision that smart metal/polymer nanocomposite particles synthesized by this green approach may find potential application in catalytic reactions because the microgel particles can not only stabilize the metal nanoparticles and retain their nanoscale properties, but also allow the switching on and off of the catalytic activity of the metal nanoparticles through controlling the accessibility of the metal nanoparticles to the reactant. Moreover, the nanocomposite particles can be easily recovered and reused. The nanocomposite particles containing bimetallic nanoparticles are of particular interest because of their possible synergistic effects [19]. For example, Ahn and co-workers have recently reported the synthesis of Ag@Au bimetallic nanoparticles on magnetic silica microspheres through seeding, coalescing, seeds to cores, and then growing shells from the cores on aminopropyl functionalized silica microspheres [20]. Xin et al. have also synthesized Au@Ag bimetallic nanoparticles using polyelectrolyte multilayers (PEMs) of poly(styrene sulfonate) (PSS) and poly(diallyldimethylammonium chloride) (PDDA) particles as support [21]. Electrostatic interaction between the cations and anions of the PEMs and metal ions was used as a driving force to attract metal ions into the system. Subsequent reduction of metal salts with reducing agents such as $\mathrm{NaBH}_{4}$ and ascorbic acid was carried out in repeated cycles to improve the loading and size of the metal nanoparticles. However, current methods for generating bimetallic nanoparticles usually involve tedious procedures that are not amenable for scale-up production. In this study, we have synthesized a dual responsive microgel particle that consists of a temperature-sensitive core of poly( $\mathrm{N}$-isopropylacryamide) and a $\mathrm{pH}$-sensitive polyethyleneimine shell.. This type of $\mathrm{pH}$ - and temperature-responsive microgel particles was used to generate silver in gold Ag@Au/polymer nanocomposite particles. The synergistic effects of the smart nanocomposite particles were studied using the reduction of $p$-nitrophenol to $p$-aminophenol as a model reaction. The controllable catalytic activity of the Au@PNIPAm/PEI nanocomposite particles was demonstrated via the tuning of solution $\mathrm{pH}$ and temperature.

\section{Materials and Methods}

\subsection{Materials}

Spindle-crystals of $\mathrm{N}$-isopropylacrylamide (NIPAM, Sigma-Aldrich, Saint Louis, MO, USA) were purified by repeated recrystallization in a mixture of toluene and $n$-hexane $(1: 5 v / v)$. Branched polyethyleneimine (PEI) with an average molecular weight of 750,000 (50 wt \% solution in water), $\mathrm{N}, \mathrm{N}$-methylenebisacrylamide (MBA) and tert-butyl hydroperoxide (TBHP, $70 \%$ aqueous solution), hydrogen tetrachloroaurate (III) trihydrate $\left(\mathrm{HAuCl}_{4} \cdot 3 \mathrm{H}_{2} \mathrm{O}\right)$, silver nitrate $\left(\mathrm{AgNO}_{3}\right)$, $p$-nitrophenol (reagent grade), and sodium borohydride were all purchased from Sigma-Aldrich Chemical Co., and used as received. Deionized water or Milli-Q water was used for dilution and dispersion medium.

\subsection{Synthesis of $A u @ P N I P A m / P E I$ Nanocomposite Particles}

The synthesis of Au-loaded poly(N-isopropyl acrylamide)/polyethyleneimine (PNIPAm/PEI) nanocomposite particles was based on our previously established method [17]. Such a method involved a simple mixing of preformed PNIPAm/PEI microgel particles with a hydrogen tetrachloroaurate (III) trihydrate $\left(\mathrm{HAuCl}_{4} \cdot 3 \mathrm{H}_{2} \mathrm{O}\right)$ solution according to the following general procedure: A stock solution of hydrogen tetrachloroaurate (III) trihydrate $\left(1.317 \times 10^{-3} \mathrm{M}\right)$ was purged with $\mathrm{N}_{2}$ for $30 \mathrm{~min}$. It was then added dropwise $(1 \mathrm{~mL})$ to the PNIPAm/PEI microgel dispersion $(20 \mathrm{~mL}, 400 \mathrm{ppm}$, molar ratio of $\mathrm{N} / \mathrm{Au}^{3+}=28$ ). The mixture was stirred at $250 \mathrm{rpm}$ for $4 \mathrm{~h}$ at $25^{\circ} \mathrm{C}$. The resultant gold-loaded microgels were purified by a single cycle of centrifugation at $12,000 \mathrm{rpm}$ and $10^{\circ} \mathrm{C}$ for $1 \mathrm{~h}$. The collected pink product was redispersed in deionized water under sonication for subsequent usage.

\subsection{Synthesis of Ag@Au/PNIPAm/PEI Nanocomposite Particles}

Synthesis of the Ag@Au bimetallic nanoparticles was carried out through a successive reduction of $\mathrm{AgNO}_{3}$ in the presence of the Au@PNIPAm/PEI particles. The preformed gold nanoparticles 
were used as seeds for the successive reduction of the silver ions to silver nanoparticles. A 1:1 molar ratio sample that contained $1 \mathrm{~mL}$ Au salt solution $\left(1.317 \times 10^{-3} \mathrm{M}\right)$ and $1 \mathrm{~mL} \mathrm{Ag}$ salt solution $\left(1.317 \times 10^{-3} \mathrm{M}\right)$ was used in this study. The reaction was first carried out at room temperature for $30 \mathrm{~min}$, followed by heating at $60^{\circ} \mathrm{C}$ for another $30 \mathrm{~min}$. During the mixing of the silver salt solution with the Au-loaded particles, the solution changed color from light pink to light gray, and eventually turned fully gray after heating the mixture.

\subsection{Catalytic Activity of Nanocomposite Particles}

The catalytic activity of both Au@PNIPAm/PEI and Ag@Au/PNIPAm/PEI nanocomposite particles were studied in an aqueous system using reduction of $p$-nitrophenol to $p$-aminophenol as a model reaction. Sodium borohydride $(200 \mu \mathrm{L}, 0.001 \mathrm{M}), p$-nitrophenol $(30 \mu \mathrm{L}, 0.001 \mathrm{M})$ and a definite quantity of the composite particles ( $40 \mu \mathrm{L}, 1.5 \mathrm{wt} \%$ solid content) were diluted to $2 \mathrm{~mL}$ with deionized water and mixed in a cuvette reactor. The reaction mixture changed from light yellow to colorless. The catalytic reaction using Au@PNIPAm/PEI (amine to gold molar ratio of 28:1) and $\mathrm{Ag} @ \mathrm{Au} / \mathrm{PNIPAm} / \mathrm{PEI}$ [amine to gold and silver ions ratio $\left(\mathrm{N} / \mathrm{Au}^{3+} \mathrm{Ag}^{+}\right)$of $28: 1$ ] were systematically studied under different solution $\mathrm{pH}$ values $(3-11)$ and temperatures $\left(25-39^{\circ} \mathrm{C}\right)$. The reduction of $p$-nitrophenol to $p$-aminophenol was monitored by the UV-visible spectroscopy (Agilent Technologies, Santa Clara, CA, USA) at a wavelength of $400 \mathrm{~nm}$ for $20 \mathrm{~min}$. Measurements were conducted at 2-min intervals.

\subsection{Measurements and Characterization}

\subsubsection{Particle Sizes and Surface Charges}

The hydrodynamic diameter and zeta-potential of the microgel templates as well as mono- and bimetallic-loaded nanocomposite particles were all measured with a Beckman Coulter Delsa Nano particle analyzer (Beckman Coulter, Brea, CA, USA) using a photon correlation spectroscopy with electrophoretic dynamic light scattering (a two-laser diode light source with a wavelength of $658 \mathrm{~nm}$ at $30 \mathrm{~mW}$ ). Hydrodynamic diameter, $D_{\mathrm{h}}$, was obtained from the Einstein Stokes equation, $D_{\mathrm{h}}=k T / 3 \pi \eta D$, where $k$ is the Boltzmann constant, $\eta$ is the dispersant viscosity, $T$ is the temperature (K), and $D$ is the diffusion coefficient. The diffusion coefficient was obtained from the decay rate of the intensity correlation function of the scattered light (i.e., correlogram), $G(\tau)=\int I(t) I(t+\tau) \mathrm{d} t$. Each measurement was carried out in triplicate. zeta-Potential measures the surface charge of particles based on their electrophoretic mobility. Samples for zeta-potential measurements were diluted to 100-200 ppm with $1 \mathrm{mM} \mathrm{NaCl}$ and measured at $25^{\circ} \mathrm{C}$.

\subsubsection{Transmission Electron Microscopy}

Transmission electron microscopy (TEM) images of monometallic gold-loaded microgels and bimetallic $(\mathrm{Ag} @ \mathrm{Au})$-loaded microgels were observed using a transmission electron microscope (JEOL 100 CX, JEOL, Tokyo, Japan) at an accelerating voltage of $100 \mathrm{kV}$. The high resolution TEM micrographs of Au and Ag@Au nanoparticles and their corresponding selected area electron diffraction (SAED) patterns were characterized by a JEOL 2010 TEM (JEOL) at an accelerating voltage of $200 \mathrm{kV}$. The sample was prepared by wetting a carbon-coated grid with a $5 \mu \mathrm{L}$ of the diluted particle dispersion, followed by drying at room temperature prior to TEM analysis. There was no pretreatment staining for all nanocomposite samples.

\subsubsection{X-ray Photoelectron Spectroscopy}

X-ray photoelectron spectroscopy (XPS) data were recorded on a multi-surface analysis system (PHI 5600, Physical Electronics, Chanhassen, MN, USA) with a monochromatic AlK $\alpha$ X-ray source (1486.6 eV). Sample spot sizes varied from 200 to $400 \mu \mathrm{m}$ in diameter. The pass energies of exciting radiations were set at 187 and $45 \mathrm{eV}$ for survey and elemental scans, respectively. The energy and 
emission currents of the electrons were $4 \mathrm{eV}$ and $0.35 \mathrm{~mA}$, respectively. Energy resolution was at $0.7 \mathrm{eV}$ with a chamber pressure of $5 \times 10^{-10}$ torr. Spectral calibration was determined by setting the $C 1 \mathrm{~s}$ component at $285.0 \mathrm{eV}$. All data acquisition was processed with a PC-based Advantage software (version 1.85, Advantage Software Co., Stuart, FL, USA). The surface composition was determined by using the manufacturer's sensitivity factors. Curve fitting of the spectrum was accomplished using a nonlinear least-squares method. A Gaussian function was assumed for the curve fitting. The deconvolution of carbon, oxygen, and nitrogen peaks was processed with MagicPlot software (version 2.5.1, MagicPlot Systems, St. Petersburg, Russia).

\subsubsection{UV-vis Spectroscopy}

UV-vis spectra were recorded on a Varian Cary 4000 Spectrophotometer using wavelengths ranging from $250-525 \mathrm{~nm}$ with an absorbance set from 0 to 1.40 a.u. Samples were diluted to appropriate concentrations and measured in the 5-mL cuvette. Actual absorbance as a function of time was plotted and a fitted curve was derived from an analysis-fitting function of Origin Pro software (v. 8.0, OriginLab Co., Northampton, MA, USA). Time-dependent UV-vis spectra (Agilent Technologies) on the reduction of $p$-nitrophenol to $p$-aminophenol using both monometallic (Au@PNIPAm/PEI) and bimetallic (Ag@Au/(PNIPAm/PEI)) nanocomposite particles as catalysts were used to monitor the catalytic reaction and calculate the catalytic constant, $k$. This constant is proportional to the catalytic rate of the reaction. UV-vis absorbance at $400 \mathrm{~nm}$ is a characteristic peak of the $p$-nitrophenol. During the course of the catalytic reaction, the intensity of this peak decreased due to its conversion to $p$-aminophenol, which appeared at wavelengths between 290 and $310 \mathrm{~nm}$. The gradual reduction in UV-vis absorbance of the $p$-nitrophenol was monitored at specific time intervals until its full disappearance.

\subsubsection{Atomic Force Microscopy}

Atomic force microscopy (AFM) images of PNIPAm/PEI microgel particles at 29 and $45{ }^{\circ} \mathrm{C}$ in aqueous solution were obtained using XE-120 inverted microscope complete AFM system with universal liquid cell option (Park Systems, Suwon, Korea). Imaging was carried out in a temperature control stage using a standard silicon nitride $\left(\mathrm{Si}_{3} \mathrm{~N}_{4}\right)$, gold-coated cantilever tip (MLCT-AUHW, Veeco, Plainview, NY, USA) in a non-contact fluid. The AFM system carries a decoupled XY-scanner with a maximum scan range of $100 \mu \mathrm{m} \times 100 \mu \mathrm{m}$. Image matrix was $256 \times 256$ pixels for fluid samples. Scan rate used was 0.5 to $0.8 \mathrm{~Hz}$ depending on the sample conditions. Humidity was adjusted from $40 \%$ to $80 \%$.

\subsubsection{Elemental Analysis of Bimetallic Nanoparticles}

The atomic percentage of individual bimetallic nanoparticle was evaluated using an Energy-dispersive Spectrometry (EDS) probe, operating in the bright field mode of a JEOL 2010 TEM. The spectral resolution was $1 \mathrm{~nm}$. In the chemical composition measurement, Au showed energy intensities at 2 and $2.6 \mathrm{keV}$, whereas $\mathrm{Ag}$ intensities were at 3 and $3.4 \mathrm{keV}$.

\section{Results and Discussion}

\subsection{Synthesis of $A u$ and $A g @ A u / P N I P A m / P E I$ Nanocomposite Particles}

The synthesis of both mono- and bimetallic nanoparticles is illustrated in Scheme 1. The gold nanoparticles were first generated through a reduction of gold salt with a polymeric amine using core-shell particles that consisted of poly( $N$-isopropyl acrylamide) cores and polyethyleneimine shells. Generation and stabilization mechanisms of gold nanoparticles and formation of Au@PNIPAm/PEI nanocomposite particles have been discussed in our previous papers $[17,18]$. Amine groups are known to have a reducing ability to generate metal nanoparticles [22]. They also can complex with metal ions and metal nanoparticles through their chelating properties $[23,24]$. When using the PNIPAm/PEI 
core-shell template, gold salt ions $\left[\mathrm{AuCl}_{4}\right]^{-}$were attracted into the template through an electrostatic interaction between the positively charged PEI shell and the negatively charged gold salt ions. The high local amine concentration could significantly enhance the reduction rate of gold salt ions to generate gold nanoparticles without the aid of any reducing agents.

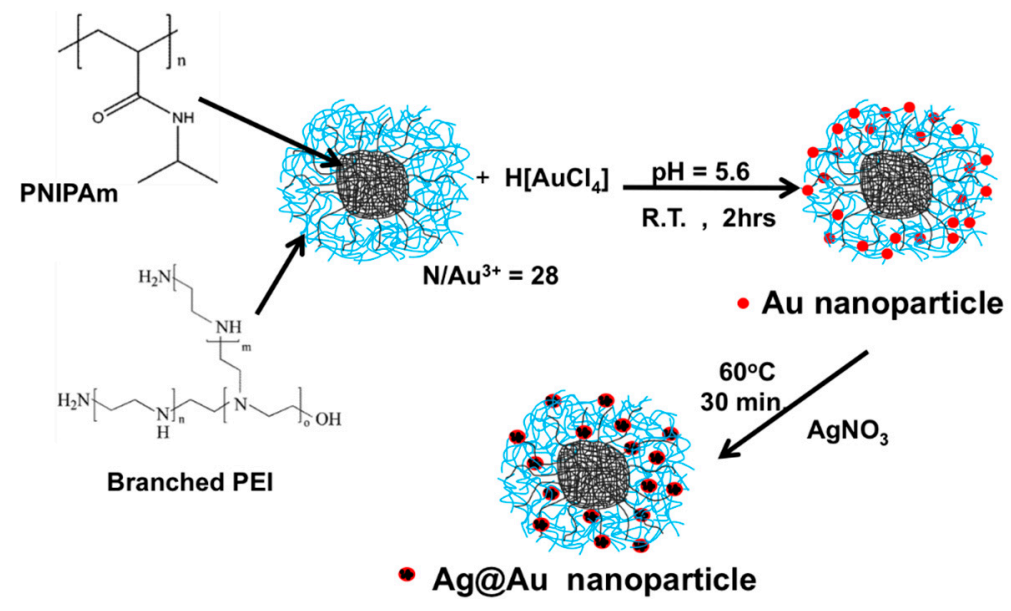

Scheme 1. Reaction scheme of the formation of $\mathrm{Au}$ and Ag@Au metal nanoparticles using PNIPAm/PEI template.

Formation of the AuNPs was evident from the change in solution color from white to light pink, which occurred after 30 to $40 \mathrm{~min}$ of reaction at room temperature. These gold nanoparticles were then used as seeds for the successive reduction of silver ions to bimetallic $\mathrm{Ag} / \mathrm{Au}$ nanoparticles using silver nitrate solution. The reduction was carried out at $60^{\circ} \mathrm{C}$ in order to increase the conversion and crystallinity of the bimetallic nanoparticles. In the presence of gold metal nanoparticles, silver ions $\left(\mathrm{Ag}^{+}\right)$could be reduced to silver nanoparticles via an under-potential deposition mechanism [25], or, as others refer to it, the noble metal induced reduction (NMIR) method [26]. In this mechanism, the gold nanoparticles acted as seeds or active sites for further growth of silver nanoparticles. The formation of silver in gold bimetallic nanoparticles was evident upon a change in the solution color from light pink to gray. This effect is attributed to the fact that the ionization potential and electron affinity values of $\mathrm{Au}$ atom are higher than those of the Ag atom. The large electronegativity value of the Au atom leads to effective charge transfer from silver to gold atoms because the second metal ion $\left(\mathrm{Ag}^{+}\right)$has lower reduction potential than gold nanoparticles $[27,28]$.

\subsection{Compositions and Morphologies of Au and Ag@Au/PNIPAm/PEI Nanocomposite Particles}

The TEM images shown in Figure 1 reveal the transformation of PNIPAm/PEI to Au and Ag@Au/PNIPAm/PEI nanocomposite particles. The PNIPAm/PEI particle displays a core-shell structure where the core has a darker contrast than the shell (Figure 1a inset). The resultant

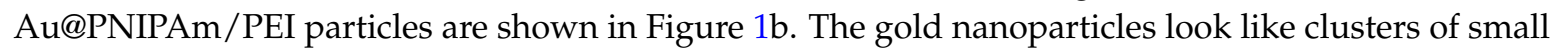
gold nanoparticles with an average diameter of $15 \pm 4.0 \mathrm{~nm}$. They were homogenously distributed within the particles. Figure 1c shows the morphology of Ag@AuPNIPAm/PEI nanocomposite particles. The bimetallic nanoparticles displayed two different intensities of contrast (Supplementary Materials Figure S1). The region in a darker shade of gray constitutes the gold nanoparticle while the lighter gray part is the silver nanoparticles. Elemental analysis results revealed that the bimetallic nanoparticles comprised 17.6 and 82.4 atomic percentage of $\mathrm{Ag}$ and $\mathrm{Au}$, respectively (Supplementary Materials Figure S2). The higher Au percentage may be attributed to the fact that only partial $\mathrm{Au}^{3+}$ ions were initially reduced to gold nanoparticles at room temperature, while the remaining gold salt ions $\left[\mathrm{AuCl}_{4}\right]^{-}$were attracted to the positively charged PEI shells. When the temperature was raised to 
$60{ }^{\circ} \mathrm{C}$, the other part of the gold salt ions was further reduced together with some $\mathrm{Ag}^{+}$ions, resulting in the formation of Ag@Au nanoparticles with a higher Au content.
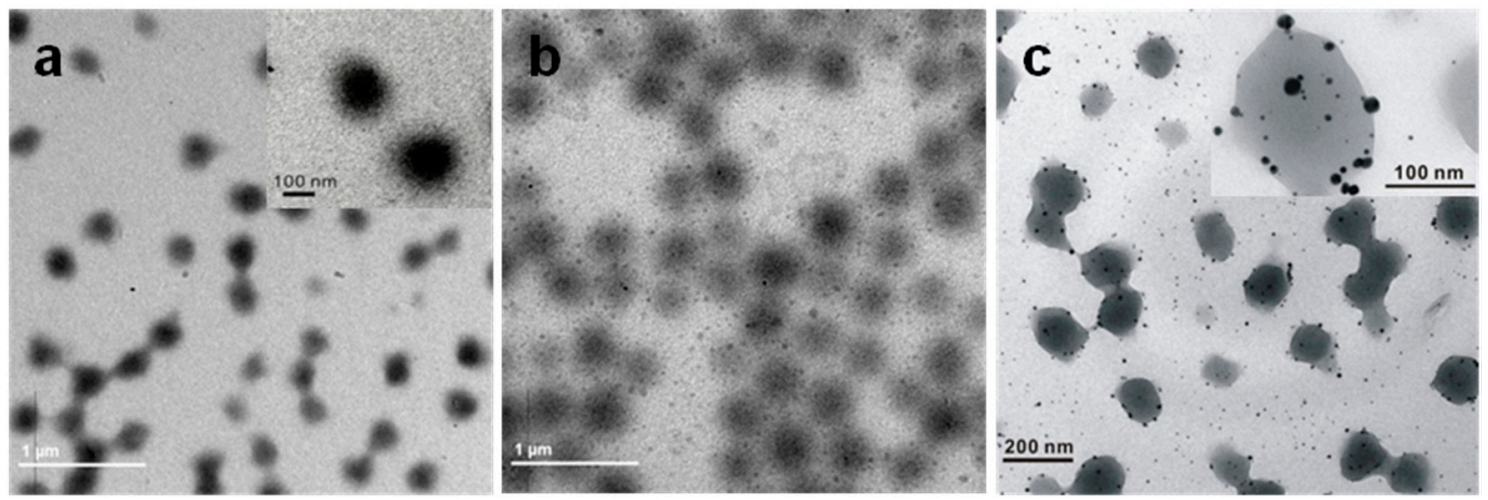

Figure 1. TEM images of (a) PNIPAm/PEI microgel templates; (b) Au/PNIPAm/PEI nanocomposite particles prepared at room temperature; (c) Ag@Au/PNIPAm/PEI nanocomposite particles formed at $60{ }^{\circ} \mathrm{C}\left(\mathrm{Au}^{3+} / \mathrm{Ag}^{+}\right.$molar ratio of $\left.1: 1\right)$.

It was noted that the average size of the bimetallic nanoparticles was only ca. $6.83 \pm 2.5 \mathrm{~nm}$ as shown in Figure 1c. Their sizes were much smaller than those of the original Au nanoclusters, indicating that the Au nanoclusters were dissociated after forming bimetallic nanoparticles. The smaller bimetallic nanoparticles were more difficult to entrap within the soft and flexible PEI shell. Thus some of them escaped from the templates and dispersed in the solution. Furthermore, the bimetallic nanoparticles located in the templates or freely dispersed in solution appeared much darker than the Au nanoclusters. This phenomenon may be attributed to the thermal treatment of the nanocomposite particles, a process that facilitates the growth of the metallic nanoparticles through further crystallization.

A high-resolution TEM (HRTEM) was performed to verify the nanostructures of mono- and bimetallic nanoparticles. In order to clearly observe the metal nanoparticles in the image, the nanocomposite particles were pre-treated with electron beam irradiation with a current density of $0.4 \mathrm{nA} / \mathrm{nm}^{2}$ for $30 \mathrm{~s}$ to partially remove the polymer template. Figure 2a shows an image of a polycrystalline Au nanostructure with a diameter of $c a .19 .1 \mathrm{~nm}$. Figure $2 \mathrm{~b}$ shows the selected area electron diffraction (SAED) pattern over several Au nanoparticles. It reveals a ring pattern indexed as (111), (200), (220), (311), and (331) of a face-centered cubic (fcc) gold lattice. Thus, the gold nanoparticle is mainly composed of (111) planes with a $d$-spacing of $0.236 \mathrm{~nm}$. The fuzzy central portion of the nanoparticle exhibited in the HRTEM image indicates that the Au nanoparticle adopts an icosahedral morphology with multiple-twinned structure [29]. Such defect is attributed to the small displacement of atoms located at the central nucleation site of the nanoparticle. Figure $2 \mathrm{c}, \mathrm{d}$ show the crystallinity and different lattice arrangement of the Ag@Au bimetallic nanoparticles, respectively. The difference in electron densities between gold and silver is due to their differences in the corresponding lattice parameter. $\mathrm{Au}(111)$ has a lattice $d$-spacing of $0.236 \mathrm{~nm}$, while that of $\mathrm{Ag}(200)$ is $0.205 \mathrm{~nm}$. Hence, the SAED analysis of the bimetallic nanoparticles further confirmed the co-existence of the crystalline orientations of $\mathrm{Au}$ and $\mathrm{Ag}$ in a single nanoparticle.

\subsection{Particle Sizes and Surface Charges of the Nanocomposite Particles}

Changes in particle sizes and surface charges of the original PNIPAm/PEI template, the $\mathrm{Au} @ \mathrm{PNIPAm} / \mathrm{PEI}$, and the Ag@Au/PNIPAm/PEI nanocomposite particles have been examined by measuring their hydrodynamic sizes and zeta-potential values. The results in Table 1 show that the introduction of gold ions into the PNIPAm/PEI template decreases both the particle size and the surface charge. The addition of $\mathrm{Ag}^{+}$ions to form bimetallic nanocomposite particles further decreases 
both the particle size and the surface charge. These effects may be attributed to the strong binding affinity of the Au or Ag@Au nanoparticles with the amine groups, resulting in the contraction of a highly swollen PEI shell. The conversion of hydrophilic amino groups to more hydrophobic moieties or crosslinking of the PEI chains due to the formation of amine radical cation during the gold ion reduction is another possible reason [22]. Despite the decrease of surface charges to almost $+5 \mathrm{mV}$, no precipitation was observed during the synthesis. However, slight aggregation of the nanocomposite particles was observed after several days of the reaction. These aggregates could be easily re-dispersed back to a stable colloidal system after sonication. Thus, the PEI and PNIPAm graft chains located in the particle shell were able to provide both electrostatic and steric stabilizations of the resultant nanocomposite particles.
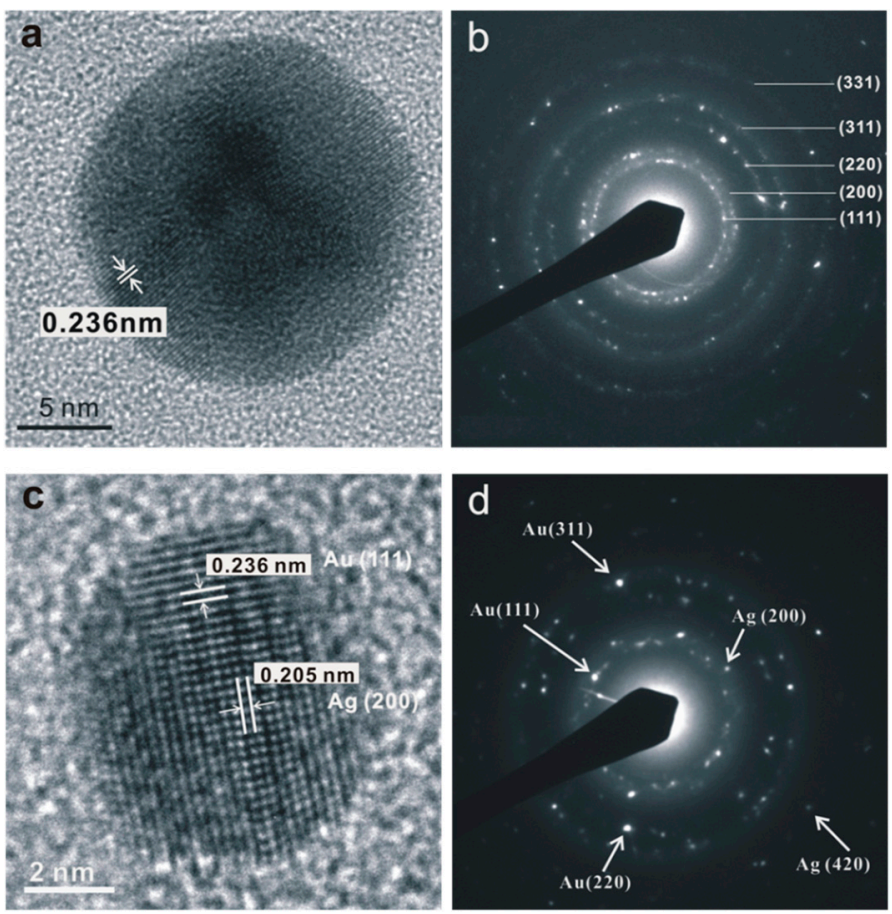

Figure 2. (a) HRTEM image of Au nanoparticle embedded within a microgel template with measured lattice $d$-spacing of $0.236 \mathrm{~nm}$; (b) the selected area electron diffraction (SAED) pattern from several Au nanoparticles; (c) HRTEM image of a magnified single Ag@Au nanocrystal with corresponding lattice parameters of $\mathrm{Au}$ and Ag; (d) SAED of Ag@Au nanoparticles.

Table 1. Particle size and zeta-potential of different nanocomposite particles.

\begin{tabular}{ccc}
\hline \multirow{2}{*}{ Type } & Mean hydrodynamic diameter & zeta-Potential \\
\cline { 2 - 3 } & $\mathbf{( n m )}$ & $\mathbf{( m V )}$ \\
\hline PNIPAm/PEI & 384 & +34 \\
$\mathrm{Au} @ P N I P A m / P E I$ & 284 & +15 \\
$\mathrm{Ag} @ \mathrm{Au} / \mathrm{PNIPAm} / \mathrm{PEI}$ & 270 & +5 \\
\hline
\end{tabular}

\subsection{Surface Chemical Composition of Nanocomposite Particles}

Surface chemical compositions of both the Au@PNIPAm/PEI and the Ag@Au/PNIPAm/PEI nanocomposite particles were characterized by XPS analysis. This technique detects elements at a depth of $10 \mathrm{~nm}$, and provides surface chemical information based on photoemission of electrons induced by X-rays. Figure 3a shows the XPS spectrum of the Au/PNIPAm/PEI nanocomposite particles. The survey spectrum reveals the characteristic binding energy peaks of $\mathrm{C}, \mathrm{O}, \mathrm{N}$, and $\mathrm{Au}$. Deconvoluted $\mathrm{C}$ 
$1 \mathrm{~s}, \mathrm{O} 1 \mathrm{~s}$, and $\mathrm{N} 1 \mathrm{~s}$ peaks are shown in Figure $\mathrm{S} 3$ in the Supplementary Materials. The deconvoluted $\mathrm{C} 1$ s peaks at 285.0 and $287.7 \mathrm{eV}$ are assigned to $\mathrm{C}-\mathrm{C} / \mathrm{C}-\mathrm{H}$ and $\mathrm{C}=\mathrm{O}$ bonds. The deconvoluted $\mathrm{O} 1 \mathrm{~s}$ peaks at 531.6 and $533.3 \mathrm{eV}$ are assigned to the amide as well as hydroxyl functional groups which come from physically absorbed $\mathrm{H}_{2} \mathrm{O}$. The deconvoluted $\mathrm{N}$ 1s peaks at 399.6 and $401.1 \mathrm{eV}$ are assigned to the nitrogen from amine and amide. The XPS elemental peak profile (Figure 3a, inset) of gold nanoparticles is confirmed based on two characteristic peaks at 84.3 and $88.0 \mathrm{eV}$, which correspond to the $\mathrm{Au} 4 f_{7 / 2}$ and $\mathrm{Au} 4 f_{5 / 2}$ of elemental gold at zero oxidation state. These results suggest that the particle shell contains components of PEI, PNIPAM and gold nanoparticles.
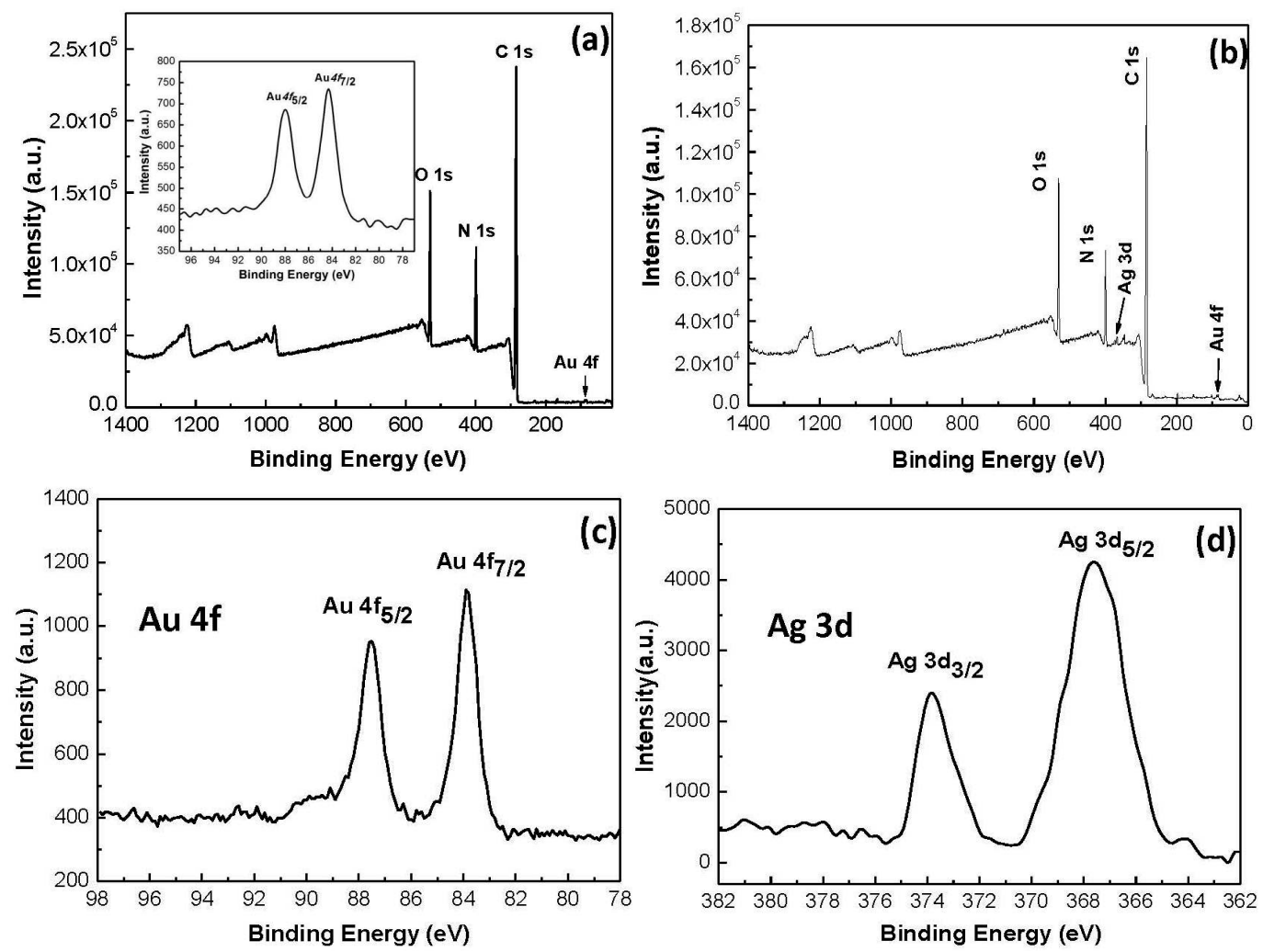

Figure 3. (a) Survey scan of Au/PNIPAm/PEI microgel. Inset is $\mathrm{Au} 4 f \mathrm{XPS}$ spectrum of $\mathrm{Au}$ nanoparticles; (b) Survey scan of Ag@AuPNIPAm/PEI microgel film (Au/Ag of 50/50 mol. ratio); (c) Au $4 f$ XPS spectrum; and (d) Ag 3d XPS spectrum of Ag@Au bimetallic nanoparticles.

Figure 3b shows the XPS spectrum of the Ag@Au/PNIPAm/PEI nanocomposite particles. The survey spectrum reveals the characteristic binding energy peaks of $\mathrm{C}, \mathrm{O}, \mathrm{N}, \mathrm{Au}$, and $\mathrm{Ag}$. Deconvoluted $\mathrm{C} 1 \mathrm{~s}, \mathrm{O} 1 \mathrm{~s}$ and $\mathrm{N} 1 \mathrm{~s}$ peaks are shown in Figure S4 in the Supplementary Materials. Figure 3c shows two peaks at binding energies of about 83.8 and $87.5 \mathrm{eV}$, which were assigned to $\mathrm{Au} 4 f_{7 / 2}$ and $\mathrm{Au} 4 f_{5 / 2}$ of zero-valent gold $\left(\mathrm{Au}^{0}\right)$, respectively. Figure $3 \mathrm{~d}$ shows the two peaks at binding energies of 367.6 and $373.8 \mathrm{eV}$, which corresponded to $\mathrm{Ag} 3 d_{5 / 2}$ and $\mathrm{Ag} 3 d_{3 / 2}$ of metallic $\mathrm{Ag}^{0}$, respectively. The differences between the $4 f_{7 / 2}$ and $4 f_{5 / 2}$ peaks for gold nanocrystal $(3.6 \mathrm{eV})$ and between the $3 d_{5 / 2}$ and $3 d_{3 / 2}$ peaks for silver nanocrystal $(\sim 6.0 \mathrm{eV})$ were of similar values of zero valent gold and silver as reported in the literature [30]. These results, in agreement with the results of the HRTEM analysis, suggest that both $\mathrm{Au}$ and $\mathrm{Ag}$ components were located in the shell region.

\subsection{Catalytic Properties of Au and Ag@Au PNIPAm/PEI Nanocomposite Particles}

The catalytic activities of the nanocomposite particles were studied through a model reaction to reduce $p$-nitrophenol to $p$-aminophenol with sodium borohydride. The reaction was monitored by a UV-vis spectrometer since $p$-nitrophenol has a characteristic maximum absorption at $400 \mathrm{~nm}$. 
A pseudo-first order kinetic reaction was chosen to derive the rate of catalytic reaction. At an excess amount of the reducing agent, $\mathrm{NaBH}_{4}\left(\right.$ mole ratio of $\mathrm{NaBH}_{4}: p$-nitrophenol = 6.7:1), the rate of reaction constant, $k$ of a pseudo-first order kinetic model can be described by Equation (1):

$$
\frac{-d_{c}}{d_{t}}=k C_{t}
$$

where the absorbance of $p$-nitrophenol at $t=0\left(A_{0}\right)$ and at $\mathrm{t}\left(A_{\mathrm{t}}\right)$ are proportional to its initial concentration $\left(C_{0}\right)$ and concentration at time $\mathrm{t}\left(C_{\mathrm{t}}\right)$, respectively. Plotting $\ln \left(C_{\mathrm{t}} / C_{\mathrm{o}}\right)$ versus time will give the rate constant based on the slope of $k\left(s^{-1}\right)$.

In a control experiment, the $p$-nitrophenol reacted only with sodium borohydride to form $p$-aminophenol as the sole product. Changes in peak intensity at $400 \mathrm{~nm}$ were monitored in intervals of every two minutes (Supplementary Materials, Figure S5). It was found that there was little change in peak intensity after up to $20 \mathrm{~min}$ of reaction, indicating a very slow reduction reaction. The rate constant derived from the slope of the linear curve was $5.4 \times 10^{-3} \cdot \mathrm{s}^{-1}$.

When Au@PNIPAm/PEI nanocomposite particles were used, the reaction proceeded approximately 4.5 times faster $\left(2.44 \times 10^{-2} \cdot \mathrm{s}^{-1}\right)$ than without the immobilized AuNPs (Figure 4a). When Ag@Au/PNIPAm/PEI nanocomposite particles were used in the same reaction system, the reaction rate was significantly enhanced, as shown in Figure $4 \mathrm{~b}$. The reduction rate constant was $6.20 \times 10^{-1} \cdot \mathrm{s}^{-1}$, indicating a much faster reaction rate than the monometallic gold nanoparticle system. Figure 5 shows the corresponding catalytic reaction rates by plotting $\ln \left(C_{t} / C_{0}\right)$ versus reaction time for the reduction of $p$-nitrophenol to $p$-aminophenol. These results indicate that the bimetallic nanocomposite particles gave a reaction 25 times faster than using monometallic gold nanocomposite particles and more than 100 times faster than the reaction without nanocomposite particles. In fact, the catalytic performance of the Ag@Au/PNIPAm/PEI nanocomposite particles was superior to other supported Ag@Au bimetallic nanoparticles reported in the literature. For examples, catalytic rate constants of a metal-organic framework-supported Au@Ag ([31], polystyrene-supported $\mathrm{Ag@Au} \mathrm{[32],} \mathrm{and} \mathrm{graphene} \mathrm{oxide} \mathrm{supported} \mathrm{Au-Ag} \mathrm{alloy} \mathrm{[33]} \mathrm{were} 4.97 \times 10^{-3} \cdot \mathrm{s}^{-1}, 15.47 \times 10^{-3} \cdot \mathrm{s}^{-1}$ and $0.05 \mathrm{~s}^{-1}$, respectively. The substantial enhancement in catalytic activity may be attributed to the synergistic effect of the bimetallic nanoparticles derived from their unique electronic and geometrical properties $[31,34,35]$. It was also suggested that the increase in the number of low coordination number edge site of Ag and corner sites of Au could enhance the catalytic activity [36].
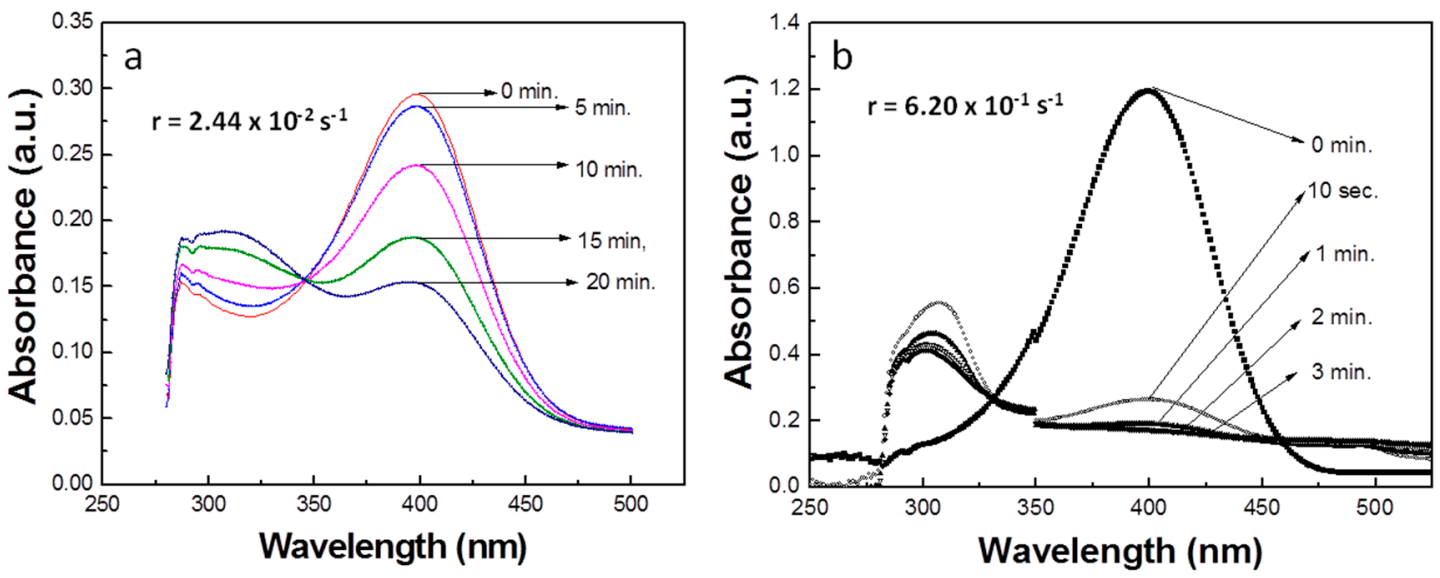

Figure 4. (a) Time-dependent UV-vis spectra on the reduction of $p$-nitrophenol to $p$-aminophenol. (a) Use of Au@PNIPAm/PEI nanocomposite particles as catalyst; (b) use of Ag@Au/PNIPAm/PEI nanocomposite particles as catalyst. 


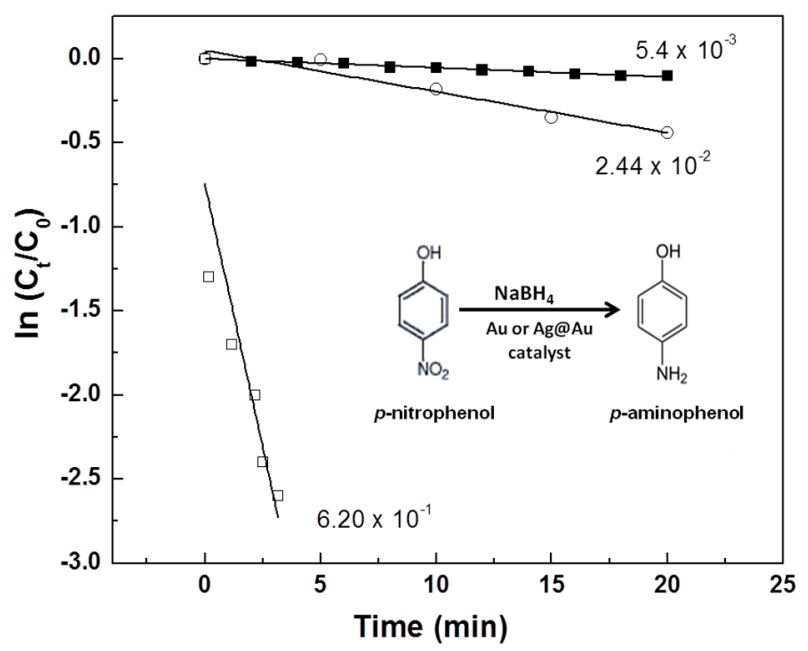

Figure 5. Plots of $\ln \left(C_{t} / C_{0}\right)$ as a function of time for the reaction at room temperature: (ם) Without catalyst; $(\bigcirc)$ In the presence of Au@PNIPAm/PEI (N/Au molar ratio = 28); ( $\square$ ) In the presence of Ag@Au PNIPAm/PEI (N/AgAu molar ratio = 28).

\subsection{Stimuli-Responsive Properties of PNIPAm/PEI Template and Tuneable Catalytic Activities of the Nanocomposite Particles}

One of the unique properties of the PNIPAm/PEI is its ability to respond to the dual stimuli of $\mathrm{pH}$ and temperature. As discussed earlier with reference to the XPS results, there were some PNIPAm chains that co-existed with PEI chains in the particle shell. To verify the morphological changes of the microgels both below and above the volume phase transition temperature (VPTT) of $32{ }^{\circ} \mathrm{C}$, we used atomic force microscopy (AFM) in a fluid mode to observe their morphologies in an aqueous solution. Figure 6a shows an image of the microgel particles at $29^{\circ} \mathrm{C}$ with particle sizes in the range of 150 to $200 \mathrm{~nm}$ and smooth surface morphology. When the temperature was raised to $45^{\circ} \mathrm{C}$, which is above the VPTT of the microgels, they became not only smaller (100-150 nm), but also had a porous surface form (Figure 6b). The particle size reduction was attributed to the shrinkage of the microgels above their VPTT. The porous surface was generated due to the contraction of the PNIPAm chains located in the shell. When the temperature was cooled down back to $29^{\circ} \mathrm{C}$, the smooth morphology and the original size of the microgel particles were restored. These results suggest that manipulation of solution temperature could induce conformational changes of the microgel with good reversibility. Thus we envisaged that the shrinking and expanding action of the nanocomposite particles could be used to control the catalytic activity of the metallic nanoparticles immobilized within the PNIPAm/PEI microgel through limiting and maximizing the exposure of the metal nanoparticles surface to reactants [37].

The effect of temperature on the catalytic activity of the Au@PNIPAm/PEI nanocomposite particles is shown in Figure 7. Catalytic activity is at the highest value at $25^{\circ} \mathrm{C}$, and decreases as temperature increases, eventually ceasing when the temperature is above $35^{\circ} \mathrm{C}$. The decrease in catalytic activity can be explained based on the accessibility of reactants to gold nanoparticles at different temperatures. When the solution temperature is below the VPTT of the Au@PNIPAm/PEI nanocomposite particles, the template swells and the gold nanoparticles become more accessible under this condition. However, when the temperature is above the VPTT of the nanocomposite particles, the PNIPAm core shrinks notably, resulting in the "dragging in" of the whole microgel particle and the covering of the catalyst surface. As a result, the reactant molecules are more difficult to penetrate and interact with the surface of gold nanocatalyst embedded in the nanocomposite particles for the reaction to proceed. The catalytic activity eventually ceased when most of the surface of the gold nanoparticles was covered by the polymer. 


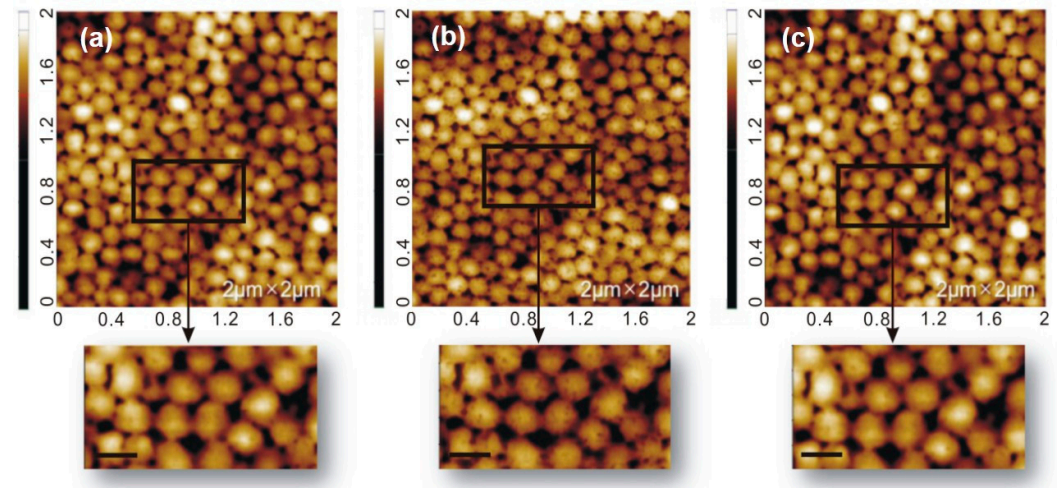

Figure 6. AFM micrographs of PNIPAM/PEI microgel particles measured in a fluid mode at different temperatures: (a) $29^{\circ} \mathrm{C}$; (b) $45^{\circ} \mathrm{C}$; and (c) cooled from 45 to $29^{\circ} \mathrm{C}$. (Scale bar: $200 \mathrm{~nm}$ ).

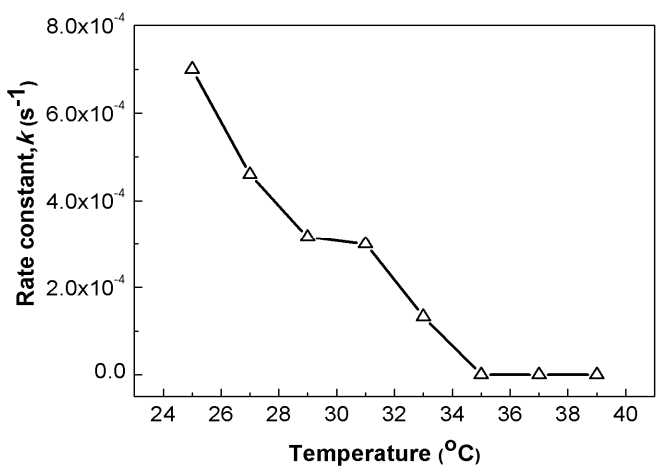

Figure 7. Effect of temperature on the rate constant of Au@PNIPAm/PEI nanocomposite particles for catalytic reduction of $p$-nitrophenol to $p$-aminophenol at $\mathrm{pH}=5.6$.

The effect of solution $\mathrm{pH}$ on the catalytic activity of the Au@PNIPAm/PEI nanocomposite particles was also systematically studied from $\mathrm{pH} 3$ to 11. Results shown in Figure 8 indicate that the highest catalytic activity is at around $\mathrm{pH} 3$ with a corresponding rate constant of $7.5 \times 10^{-3} \cdot \mathrm{s}^{-1}$. This activity is almost 10 times higher than at the neutral $\mathrm{pH}\left(7.4 \times 10^{-4} \cdot \mathrm{s}^{-1}\right)$. An abrupt reduction in catalytic activity was found in the $\mathrm{pH}$ range of 3.0-3.5. Further increasing the solution $\mathrm{pH}$ to 7.5 has little influence on the catalytic activity. When increasing the $\mathrm{pH}$ to 11 , its catalytic activity ceases. To ensure that the acid-catalyzed reaction was minimal or had no effect at all, a control experiment was performed at $\mathrm{pH} 3$ in the absence of nanocomposite particles. The reduction rate of $p$-nitrophenol to $p$-aminophenol at $\mathrm{pH} 3$ was found to be $1.52 \times 10^{-5} \cdot \mathrm{s}^{-1}$. This value is much smaller than the reaction using $\mathrm{Au} / \mathrm{PNIPAm} / \mathrm{PEI}$ nanocomposite particles (reaction rate was $7.5 \times 10^{-3} \cdot \mathrm{s}^{-1}$ ) at the same $\mathrm{pH}$ of 3 . The results confirmed that an enhanced reaction rate at $\mathrm{pH} 3$ was not caused by the acid-catalyzed reaction.

The variation of catalytic activity may be attributed to the $\mathrm{pH}$-responsiveness of the PEI shell of the nanocomposite template. It is known that the percentage of protonated amines varies with the solution $\mathrm{pH}$ [38]. For example, percentages of protonated amines at $\mathrm{pH} 3,7$, and 9 are around $75 \%, 25 \%$, and $8 \%$, respectively. Therefore, the effect of solution $\mathrm{pH}$ on the catalytic activity of the $\mathrm{Au} @ \mathrm{PNIPAm} / \mathrm{PEI}$ nanocomposite particles may be explained by the following reasons: Under low acidic $\mathrm{pHs}(\mathrm{pH}=3-4)$, a high protonation degree of the amino groups occurs, resulting in the increase of charge density and the stretching of the PEI network. The expanded PEI shell provides more exposure for the gold nanoparticle to interact with reactant molecules. On the other hand, increasing the solution $\mathrm{pH}$ leads to lowering the protonation degree, thus forming a more compacted PEI shell. Consequently, the shielding of the PEI shell makes it difficult for the reacting species to diffuse into 
the catalytic surface of the gold nanoparticles. These results demonstrate that the catalytic activity of Au@PNIPAm/PEI nanocomposite particles can be easily turned "on" and "off" by adjusting the solution $\mathrm{pH}$.

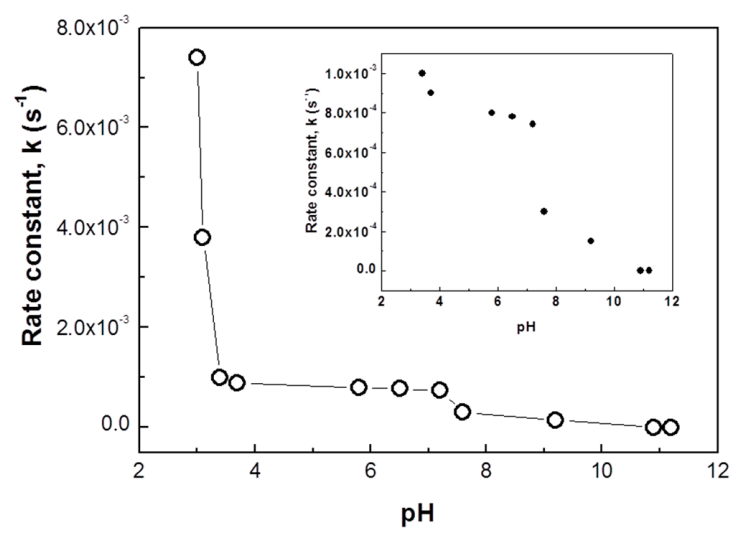

Figure 8. Effect of $\mathrm{pH}$ on the rate constant of Au@PNIPAm/PEI nanocomposite particles for catalytic reduction of $p$-nitrophenol to $p$-aminophenol at room temperature.

\subsection{Reusability of Nanocomposite Particles}

The reusability of $\mathrm{Au} / \mathrm{PNIPAm} / \mathrm{PEI}$ nanocomposite particles has been examined by comparing morphologies of nanocomposite particles before and after the catalytic reactions. The $\mathrm{Au} / \mathrm{PNIPAm} / \mathrm{PEI}$ nanocomposite particles were recovered after one cycle of catalytic reaction through centrifugation and redispersion. TEM images shown in Figure 9 reveal that the morphology of the recovered nanocomposite particles is quite similar to those original nanocomposite particles. These results suggest that the nanocomposite particles may be reusable for catalytic reaction.
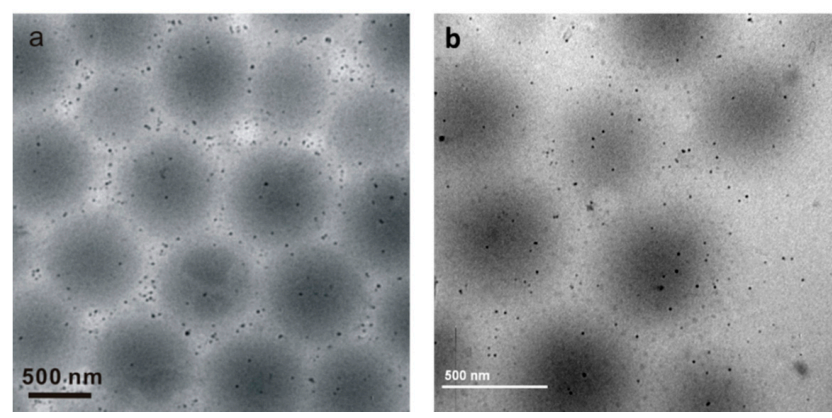

Figure 9. TEM images (a) before catalysis and (b) after catalysis. The Au/PNIPAm/PEI nanocomposites was recovered at 10,000 rpm centrifugation for 1 hour, followed by redispersing the particles in deionized water.

\section{Conclusions}

We have developed a simple route for in situ synthesis of Au and Ag@Au nanoparticles using smart PNIPAm/PEI core-shell microgel particles as dual reductant and template. The gold nanoparticles were initially formed through a reduction of gold ions with highly concentrated amine functional groups of the microgel. The resultant gold nanoparticles were then used as seeded nanoparticles for further reduction of silver ions to form bimetallic alloy nanoparticles. The use of polymeric amine-based particles to produce metal/polymer nanocomposite particles in an aqueous solution is a simple and green synthesis without using any organic solvent, reducing or stabilizing agents. The resulting nanocomposite particles possess not only excellent catalytic activities with good reproducibility and stability, but also smart properties that allow the tuning of catalytic activities of the 
metal nanoparticles through varying solution $\mathrm{pH}$ and temperature. Therefore, these metal nanoparticle immobilized nanocomposite particles possess high potential for practical applications in catalysis.

Supplementary Materials: The supplementary materials can be found at www.mdpi.com/2073-4360/ $8 / 4 / 105 /$ s1.

Acknowledgments: We gratefully acknowledge the Hong Kong Polytechnic University for its financial support of this research and Park Systems, Suwon, Korea for the AFM measurement.

Author Contributions: Noel Peter Bengzon Tan and Pei Li conceived and designed the experiments; Noel Peter Bengzon Tan accomplished the preparation of metal/polymer nanocomposite particles, measurements of particle size, surface charge, and UV spectroscopic study of the catalytic activity of the nanocomposite particles. Cheng Hao Lee performed TEM and XPS analysis; Pei Li, Noel Peter Bengzon Tan, and Cheng Hao Lee analyzed the results and wrote the manuscript.

Conflicts of Interest: The authors declare no conflict of interest.

\section{References}

1. Mody, V.V.; Siwale, R.; Singh, A.; Mody, H.R. Introduction to metal nanoparticles. J. Pharm. Bioall. Sci. 2010, 2, 282-289. [CrossRef] [PubMed]

2. Huang, D.; Yang, G.; Feng, X.; Lai, X.; Zhao, P. Triazole-stabilized gold and related noble metal nanoparticles for 4-nitrophenol reduction. New J. Chem. 2015, 39, 4685-4694. [CrossRef]

3. Ruíz-Baltazar, A.; Esparza, R.; Rosas, G.; Pérez, R. Effect of the surfactant on the growth and oxidation of iron nanoparticles. J. Nanomater. 2015. [CrossRef]

4. Hu, J.; Yang, Q.; Yang, L.; Zhang, Z.; Su, B.; Bao, Z.; Ren, Q.; Xing, H.; Dai, S. Confining noble metal $(\mathrm{Pd}, \mathrm{Au}, \mathrm{Pt})$ nanoparticles in surfactant ionic liquids: Active non-mercury catalysts for hydrochlorination of acetylene. ACS Catal. 2015, 5, 6724-6731. [CrossRef]

5. Murugadoss, A.; Chattopadhyay, A. A “Green” chitosan-silver nanoparticle composite as a heterogeneous as well as micro-heterogeneous catalyst. Nanotechnology 2008, 19, 15603-15611. [CrossRef] [PubMed]

6. Wang, Z.; Tan, B.; Hussain, I.; Schaeffer, N.; Wyatt, M.F.; Brust, M.; Cooper, A.I. Design of polymeric stabilizers for size-controlled synthesis of monodisperse gold nanoparticles in water. Langmuir 2007, 23, 885-895. [CrossRef] [PubMed]

7. Bingwa, N.; Meijboom, R. Evaluation of catalytic activity of Ag and Au dendrimer-encapsulated nanoparticles in the reduction of 4-nitrophenol. J. Mol. Catal. 2015, 396, 1-7. [CrossRef]

8. Gopalan, P.R. Cyclodextrin-stabilized metal nanoparticles: Synthesis and characterization. Int. J. Nanosci. 2010, 9, 487-494. [CrossRef]

9. Biffis, A.; Orlandi, N.; Corain, B. Microgel-stabilized metal nanoclusters: Size control by microgel nanomorphology. Adv. Mater. 2003, 15, 1551-1555.

10. Varsha, T.; Namdeo, M.; Mohan, Y.M.; Bajpai, S.K.; Bajpai, M. Review on polymer, hydrogel and microgel metal nanocomposites: A facile nanotechnological approach. J. Macromol. Sci. Part A 2008, 45, 107-119.

11. Karg, M.; Hellweg, T. New "smart" poly(NIPAM) microgels and nanoparticle microgel hybrids: Properties and advances in characterization. Curr. Opin. Colloid Interface Sci. 2009, 14, 438-450. [CrossRef]

12. Strong, L.E.; West, J.L. Thermally responsive polymer-nanoparticles composites for biomedical application. WIREs Nanomed. Nanobiotechnol. 2011, 3, 307-317. [CrossRef] [PubMed]

13. Plaza, H. Antimicrobial polymers with metal nanoparticles. Int. J. Mol. Sci. 2015, 16, 2099-2116. [CrossRef] [PubMed]

14. Han, D.; Zhang, Q.M.; Serpe, M.J. Poly( $N$-isopropylacrylamide)-co-(acrylic acid) microgel/Ag nanoparticles hydrides for the colorimetric sensing of $\mathrm{H}_{2} \mathrm{O}_{2}$. Nanoscale 2015, 7, 2784-2789. [CrossRef] [PubMed]

15. Ballauff, M.; Lu, Y. Smart nanoparticles: Preparation, characterization and applications. Polymer 2007, 48, 1815-1823. [CrossRef]

16. Lu, Y.; Mei, Y.; Drechsler, M.; Baffauff, M. Thermosensitive core-shell particles as carriers for Ag nanoparticles: Modulating the catalytic activity by a phase transition in networks. Angew. Chem. Int. Ed. 2006, 45, 813-816. [CrossRef] [PubMed]

17. Tan, N.P.B.; Lee, C.H.; Chen, L.; Ho, K.M.; Lu, Y.; Ballauff, M.; Li, P. Facile synthesis of gold/polymer nanocomposite particles using polymeric amine-based particles as dual reductants and templates. Polymer 2015, 76, 271-279. [CrossRef] 
18. Tan, N.P.B.; Lee, C.H.; Li, P. Influence of temperature on the formation and encapsulation of gold nanoparticles using a temperature-sensitive template. Data Brief. 2015, 5, 434-438. [CrossRef] [PubMed]

19. Sankar, M.; Dimitratos, N.; Miedziak, P.J.; Wells, P.P.; Kiely, C.J.; Hutchings, G.J. Designing bimetallic catalysts for a green and sustainable future. Chem. Soc. Rev. 2012, 41, 8099-8139. [CrossRef] [PubMed]

20. Park, H.H.; Woo, K.; Ahn, J.P. Core-shell bimetallic nanoparticles robustly fixed on the outermost surface of magnetic silica microspheres. Sci. Rep. 2013, 3, 1497-1503. [CrossRef] [PubMed]

21. Zhang, X.; Su, Z. Polyelectrolyte-multilayer-supported Au@Ag core-shell nanoparticles with high catalytic activity. Adv. Mater. 2012, 24, 4574-4577. [CrossRef] [PubMed]

22. Newman, J.D.S.; Blanchard, G.J. Formation of gold nanoparticles using amine reducing agents. Langmuir 2006, 22, 5882-5887. [CrossRef] [PubMed]

23. Zelewsky, A.V.; Barbosa, L.; Schläpfer, C.W. Poly(ethylenimines) as Brønsted bases and as ligands for metal ions. Coord. Chem. Rev. 1993, 123, 229-246. [CrossRef]

24. Kobayashi, S.; Hiroshi, K.; Tokunoh, M.; Saegusa, T. Chelating properties of linear and branched poly(ethylenimines). Macromolecules 1987, 20, 1496-1500. [CrossRef]

25. Herrero, E.; Buller, L.J.; Abruna, H.D. Underpotential deposition at single crystal surfaces of Au, Pt, Ag and other materials. Chem. Rev. 2001, 101, 1897-1930. [CrossRef] [PubMed]

26. Wang, D.; Li, Y. One-pot protocol for Au-based hybrid magnetic nanostructures via a noble-metal-induced reduction process. J. Am. Chem. Soc. 2010, 132, 6280-6281. [CrossRef] [PubMed]

27. Cheng, L.C.; Huang, J.H.; Chen, H.M.; Lai, T.C.; Yang, K.Y.; Liu, R.S.; Hsiao, M.; Chen, C.H.; Her, L.J.; Tsai, D.P. Seedless, silver-induced synthesis of star-shaped gold/silver bimetallic nanoparticles as high efficiency photothermal therapy reagent. J. Mater. Chem. 2012, 22, 2244-2253. [CrossRef]

28. Cuenya, B.R.; Baeck, S.H.; Jaramillo, T.F.; McFarland, E.W. Size- and support-dependent electronic and catalytic properties of $\mathrm{Au}^{0} / \mathrm{Au}^{3+}$ nanoparticles synthesized from block copolymer micelles. J. Am. Chem. Soc. 2003, 125, 12928-12934. [CrossRef] [PubMed]

29. Buffat, P.A.; Flueli, M.; Spycher, R.; Stadelmann, P.; Borel, J.P. Crystallographic structure of small gold particles studied by high-resolution electron microscopy. Faraday Discuss. 1991, 92, 173-187. [CrossRef]

30. Shanmugam, S.; Viswanathan, B.; Varadarajan, T.K. Photochemically reduced polyoxometalate assisted generation of silver and gold nanoparticles in composite films: A single step route. Nanoscale Res. Lett. 2007, 2, 175-183. [CrossRef]

31. Jiang, H.L.; Akita, T.; Ishida, T.; Haruta, M.; Xu, Q.J. Synergistic catalysis of Au@Ag core-shell nanoparticles stabilized on metal-organic framework. J. Am. Chem. Soc. 2011, 133, 1304-1306. [CrossRef] [PubMed]

32. Zhang, S.; Wu, W.; Xiao, X.; Zhou, J.; Xu, J.; Ren, F.; Jiang, C. Polymer-supported bimetallic Ag@AgAu nanocomposites: Synthesis and catalytic properties. Chem. Asian J. 2012, 7, 1781-1788. [CrossRef] [PubMed]

33. Wu, T.; Ma, J.; Wang, X.; Liu, Y.; Xu, H.; Gao, J.; Wang, W.; Liu, Y.; Yan, J. Graphene oxide supported Au-Ag alloy nanoparticle with different shapes and their high catalytic activities. Nanotechnology 2013, 24, 125301. [CrossRef] [PubMed]

34. Wang, A.Q.; Liu, J.H.; Lin, S.D.; Lin, T.S.; Mou, C.Y. A novel efficient Au-Ag alloy catalyst system: Preparation, activity, and characterization. J. Catal. 2005, 233, 186-197. [CrossRef]

35. Benkó, T.; Beck, A.; Frey, K.; Srankóa, D.F.; Geszti, O.; Sáfrán, G.; Marótic, B.; Schay, Z. Bimetallic $\mathrm{Ag}-\mathrm{Au} / \mathrm{SiO} 2$ catalysts: Formation, structure and synergistic activity in glucose oxidation. Appl. Catal. A Gen. 2014, 479, 103-111. [CrossRef]

36. Back, S.; Yeom, M.S.; Jung, Y.S. Active sites of Au and Ag nanoparticle catalysts for $\mathrm{CO}_{2}$ electroreduction to CO. ACS Catal. 2015, 5, 5089-5096. [CrossRef]

37. Kawaguch, H. Thermoresponsive microhydrogels: Preparation, properties and applications. Polym. Int. 2014, 63, 925-932. [CrossRef]

38. Suh, J.; Lee, S.H.; Kim, S.M.; Hah, S.S. Conformational flexibility of poly(ethyleneimine) and its derivative. Bioorg. Chem. 1997, 25, 221-231. [CrossRef]

(C) 2016 by the authors; licensee MDPI, Basel, Switzerland. This article is an open access article distributed under the terms and conditions of the Creative Commons by Attribution (CC-BY) license (http:/ / creativecommons.org/licenses/by/4.0/). 